\title{
MIMO performance of a planar logarithmically periodic antenna with respect to measured channel matrices
}

\author{
H. Rabe ${ }^{1}$, D. Kornek ${ }^{1}$, M. Stege ${ }^{2}$, and I. Rolfes ${ }^{1}$ \\ ${ }^{1}$ Institute for Radiofrequency and Microwave Engineering, Hanover, Germany \\ ${ }^{2}$ Signalion GmbH, Dresden, Germany
}

\begin{abstract}
The increasing interest in wireless transmission of highest data rates for multimedia applications (e.g. HDTV) demands the use of communication systems as e.g. described in the IEEE $802.11 \mathrm{n}$ draft specification for WLAN including spatial multiplexing or transmit diversity to achieve a constant high data rate and a small outage probability. In a wireless communications system the transmission of parallel data stream leads to multiple input/multiple output (MIMO) systems, whose key parameters heavily depend on the properties of the mobile channel. Assuming an uncorrelated channel matrix the correlation between the multiplexed data streams is caused by the coupling of the antennas, so that the radiation element becomes an even more important part of the system. Previous work in this research area (Klemp and Eul, 2006) has shown that planar log.-per four arm antennas are promising candidates for MIMO applications providing two nearly decorrelated radiators, which cover a wide frequency range including both WLAN bands at $2.4 \mathrm{GHz}$ and $5.4 \mathrm{GHz}$. Up to now the MIMO performance of this antenna is mainly analyzed by simulations. In this contribution measured channel matrices in a real office environment are studied in terms of the antenna's MIMO performance such as outage probability. The obtained results recorded by using a commercial platform are compared to the simulated ones.
\end{abstract}

\section{Introduction}

The possibility of achieving a remarkable performance gain of data rate and link reliability by using spatial multiplexing in wireless communication systems lead to the first WLAN standard 802.11n that uses MIMO techniques. However, there are no concrete rules for antenna designers to gener-

Correspondence to: H. Rabe

(rabe@hft.uni-hannover.de) ate antennas that offer a good MIMO performance preferably in as many channel scenarios as possible. The used antenna structures, the placing as well as the arrangement can have significant influence on the overall system performance. This contribution shows, how polarization diversity can be used to increase orthogonality of the single subchannels from a theoretical point of view by means of a stochastic channel model together with a narrow band assumption (see Sect. 2) for a $2 \times 2$ MIMO system. To prove this statement several measurements of channel matrices have been made in an office environment with planar logarithmically periodic antennas (LPs) as well as monopole antennas at typical WLAN frequencies at $2.4 \mathrm{GHz}$ and $5.4 \mathrm{GHz}$. The measurements have been taken with the HaLo220 testbed that allows the transmission and reception of two RF signals simultaneously. A LP antenna enables the radiation of two linearly polarized waves with an axial ratio of better than $30 \mathrm{~dB}$. The performance differences between a setup with LP antennas and with standard monopole antennas are discussed in Sect. 3.2. The paper ends with a conclusion.

\section{MIMO channel}

To make reliable MIMO measurements of channel matrices some general boundaries should be taken into account. Sections 2.1 and 2.2 describe the necessity of considering the coherence time and the coherence bandwidth in order to fulfil the narrow band assumption and to preserve stationarity of the channel for short time periods. By considering the aforementioned conditions the channel can be expressed as a channel matrix $\mathbf{H}$ consisting of complex values shown in Sect. 2.3. This section further discusses the influence of the antennas on the channel matrix separately for LOS (Line Of Sight) and NLOS (Non Line Of Sight) scenarios.

Published by Copernicus Publications on behalf of the URSI Landesausschuss in der Bundesrepublik Deutschland e.V. 


\subsection{Coherence time}

In general, the impulse response of a radio channel is assumed to be linear but not stationary. The movement of persons and objects in the channel causes more or less rapidly changing wave propagation conditions. In the case of a WLAN system, which is intended to operate in an indoor environment, the velocity of the moving scatterers rarely exceeds $v_{s c}=5 \mathrm{~km} / \mathrm{h}$ (Jiménez, 2002) in most cases. Depending on the center frequency the phase of the transmission path is being changed due to a moving scatterer. Regarding a short time period only, the transmission phase can be assumed to be stationary as well as the whole channel impulse response. The time interval, in which the correlation of the channel response does not rise above 0.5 is defined as coherence time $T_{C}$ in this paper. During the coherence time interval $T_{C}$ the channel impulse response can be described as one complex delta peak that scales the obtained signal in the equivalent baseband domain. As a rule of thumb formula Eq. (1) can be used to determine $T_{C}$.

$T_{C}=\frac{v_{s c}}{\lambda}$

The coherence time decreases with shrinking wavelength so the worst case is a transmission at the highest used frequency. For the high WLAN frequency band around $5.4 \mathrm{GHz}$ the coherence time can be estimated to $T_{C}=40 \mathrm{~ms}$. However, this formula is not adequate in many cases. Another approach (Rappaport, 1996), that is said to be more appropriate determines a smaller value of $T_{C}=18 \mathrm{~ms}$ which was assumed to be valid for the examined WLAN channels.

\subsection{Coherence bandwidth}

It is furthermore assumed, that every frequency component in the baseband signal is distorted the same way so the frequency response of the channel is ideally flat. It is important for accurate measurements that this requirement is fulfilled by the radiated signals. Analogous to the coherence time one can determine a coherence bandwidth $B_{C}$ which is directly connected to the delay spread $\sigma_{\tau}$ of the channel. The longer the dominant propagation pathes are the more sensitive the phase difference is between adjacent signal frequencies. The coherence bandwidth can be estimated by expression (2) according to Sklar (2001) and is related to the delay spread $\sigma_{\tau}$ that includes the path lengths of the channel implicitly.

$B_{C}=\frac{1}{50 \sigma_{\tau}}$

For indoor channels the delay spread strongly depends on the scenario. When transmitter and receiver are both situated in a small room, where the path lengths are supposed to be short, there will be no large spread in the arrival of the signal echoes. Even if the path length are long the delay spread can be short if a strong line of sight component or another dominant propagation path exists. A worst case scenario would be a channel with two similarly weighted pathes of different length, for example a LOS and a reflected component coming from a wall. In this paper the delay spread is assumed to stay below $\sigma_{\tau}=100 \mathrm{~ns}$, which is more than sufficient for the office environment where the measurement took place and about the double of the value measured by McDonnel et al. (1998). Therefore, the coherence bandwidth can be estimated from expression (2) to $200 \mathrm{kHz}$. This restriction will be considered in Sect. 3.

\subsection{MIMO channel matrix}

With the preceding simplifications of a narrow band channel the impulse response $h(t)$ degenerates to one complex value between transmit and receive antenna within a coherence time interval. With multiple transmit and receive antennas each single impulse response value can be composed in the MIMO matrix $\mathbf{H}$ as shown in Fig. 1. The receiver signal expands to a receive vector $\boldsymbol{y}$, which $M$ elements are a linear combination of the $N$ elements in the transmit vector $s$. The relation between transmit and receive vectors is expressed in Eq. (3).

$$
\left(\begin{array}{c}
y_{1} \\
y_{2} \\
\vdots \\
y_{m}
\end{array}\right)=\left(\begin{array}{cccc}
h_{11} & h_{12} & \ldots & h_{1 n} \\
h_{21} & h_{22} & \ldots & h_{2 n} \\
\vdots & \vdots & \ddots & \vdots \\
h_{m 1} & h_{m 2} & h_{m 3} & h_{m n}
\end{array}\right) \cdot\left(\begin{array}{c}
s_{1} \\
s_{2} \\
\vdots \\
s_{n}
\end{array}\right)
$$

The channel capacity $C_{\mathrm{MIMO}}$ in the case of equally divided power $P_{T}$ over the transmit antennas can be expressed as follows according to Foschini and Gans (1998).

$$
\begin{aligned}
C_{\mathrm{MIMO}} & =\log _{2} \operatorname{det}\left(\overline{\mathbf{E}}-\frac{P_{T}}{M \sigma_{n}^{2}} H H^{H}\right) \\
\Leftrightarrow C_{\mathrm{MIMO}} & =\sum_{i} \log _{2}\left(1+\frac{P_{T}}{M \sigma_{n}^{2}} \lambda_{i}\right)
\end{aligned}
$$

$\overline{\mathbf{E}}$ is the identity matrix, $\lambda_{i}$ is the $i$ th eigenvalue of the matrix product between $H$ and its hermitian $H^{H}$ and $\sigma_{n}^{2}$ is the noise power which is assumed to be white gaussian noise. From Eq. (4) one can see that the spectral efficiency is increased by using higher transmit power or by trying to achieve a high rank of the matrix product $H H^{H}$ or the matrix $\mathbf{H}$, respectively. For WLAN indoor channels rayleigh fading is assumed so the matrix $\mathbf{H}$ is filled with independent and identically-distributed (IID) complex random variables for each coherence time. Unfortunately it is very hard to achieve the data rate which is offered by the pure rayleigh fading channel model. In fact the signals are transmitted and received at the feeding points of the antennas and will be somehow correlated (see Schumacher and Kermoal, 2002) which avoids reaching the theoretical limit. The model which is used in $802.11 \mathrm{n}$ for modeling some of these effects is a stochastic channel model with one or more azimuthal probability density functions describing the incoming and outgoing waves from an antenna array for a few coherence times. 


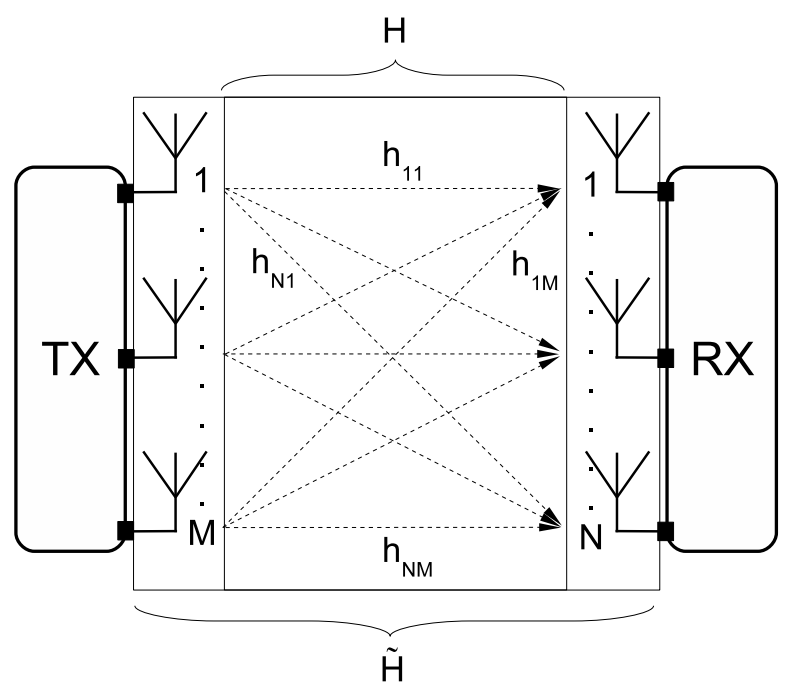

Fig. 1. The MIMO channel matrix $\mathbf{H}$ itself and the matrix $\tilde{\mathbf{H}}$ including correlation due to the involved antennas.

Considering the resulting correlation of the signals on the feeding point extends the channel matrix $\mathbf{H}$ to the matrix $\tilde{\mathbf{H}}$ as presented in Fig. 1. In addition to the stochastic modeling of the WLAN channel the official model considers a LOS component which is added to the pure stochastic channel. Therefore the channel matrix $\tilde{\mathbf{H}}$ can be thought of being composed from a sum of two matrices $\tilde{\mathbf{H}}_{\text {LOS }}$ and $\tilde{\mathbf{H}}_{\mathrm{NLOS}}$ with different weights as expressed in Eq. (5).

$\tilde{\mathbf{H}}=\sqrt{P} \cdot\left(\sqrt{\frac{K}{K+1}} \tilde{\mathbf{H}}_{\mathrm{LOS}}+\sqrt{\frac{1}{K+1}} \tilde{\mathbf{H}}_{\mathrm{NLOS}}\right)$

The rice factor $K$ determines these weights and is fixed according to a certain channel scenario while the NLOS component is following the fluctuations of the channel modeled by IID values. The power $P$ describes the power of the transmitted signals and scales the whole channel matrix. For an antenna designer it is desirable to benefit from spatial multiplexing offered by the NLOS and LOS component of the channel as well. The following two Sects. 2.3.1 and 2.3.2 describe which antenna parameters are important to look at in order to increase the channel capacity.

\subsubsection{Antennas in a LOS scenario}

The LOS component describes a fixed propagation path between transmit and receive antenna. This fact prevents the use of pattern diversity (see Sect. 2.3.2) in this case, which aims at exciting the eigenpathes of the channel using different propagation pathes. A more promising approach is the use of polarization diversity. Radiating and receiving two orthogonally polarized waves with a low crosstalk allows the transmission of two independent data channels. Figure 2 reveals this relation. A transmit antenna radiates an ideally

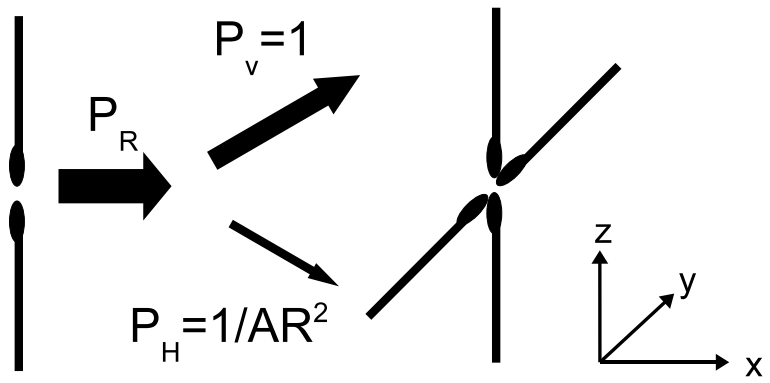

Fig. 2. Reception of an ideally linearly polarized wave by a cross dipole with finite axial ratio.

vertical polarized wave with the power $P_{R}$. The receiving dipole antennas are oriented along the z- and the y-axis, respectively. In general, the antennas are coupled, so the horizontally placed dipole in the $\mathrm{x}-\mathrm{y}$ plane will receive a little amount of the vertical polarized wave anyway. This property is described by the axial ratio $A R$, which is expressed as the ratio between the maximum and the minimum absolute value of the electric field strengths in the polarization ellipse. Assigning a certain $A R$ to the receiving antennas in Fig. 2 and normalizing the vertically received power $P_{H}$ to 1 the horizontally received can be determined to $P_{H}=1 / A R^{2}$. In this symmetrical case, the same relation can be shown in the case of receiving a horizontal wave with the vertically oriented dipole, where the received power is also determined by means of the $A R$. The higher the $A R$ is, the higher the suppression of the unwanted orthogonal polarization direction is. According to the channel scenario in Fig. 2 the channel matrix $\mathbf{H}$ for the $2 \times 2$ case of infinite $A R$ on transmitter side and a finite $A R$ on receiver side, the channel matrix is proportional to expression (6).

$\tilde{\mathbf{H}}_{\mathrm{LOS}} \approx\left(\begin{array}{cc}1 & 1 / A R \\ 1 / A R & 1\end{array}\right)$

The equation shows that the channel matrix approaches more and more the identity matrix as the $A R$ increases. The eigenvalues of $\tilde{H} \tilde{H}^{H}$ and the spectral efficiency, respectively, are increasing similarly. If an antenna array offers a high $A R$ over a wide angular spread, the channel matrix rank will stay high for many LOS cases.

\subsubsection{Antennas in a NLOS scenario}

For the NLOS components of the channel it is necessary to unite the stochastic channel model including angle of arrival (AoA), angle of departure (AoD) and the according angular spreads with the complex far field patterns of the single antenna elements in the array. The established model in (Schumacher and Kermoal, 2002) describes a way to include the antenna influences by stamping a correlation on the elements of the pure channel matrix $\mathbf{H}$ resulting in the matrix $\tilde{\mathbf{H}}$. The 


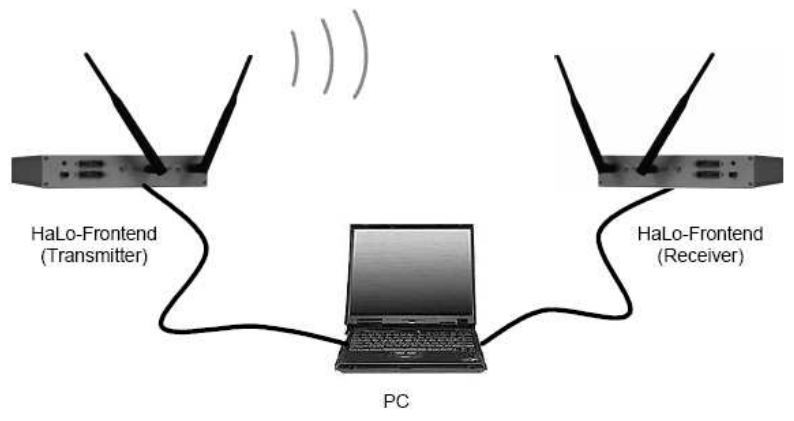

Fig. 3. The HaLo220 MIMO prototyping system.

envelope correlation coefficient $\rho_{e}$ can be approximated by the square of the absolute value of the correlation coefficient $\left|\rho_{i j}\right|^{2}$ between the antennas $i$ and $j$ in an antenna array. It determines the degree of linear independency of the columns in the channel matrix and is expressed in formula 7 according to Fujimoto and James.

$\rho_{e}=\left|\rho_{i j}\right|^{2}=\frac{\left|R_{i j}\right|^{2}}{\sigma_{i}^{2} \cdot \sigma_{j}^{2}}$

The variance $\sigma^{2}$ is the part of the radiated or received power which contributes to the transmission in a certain channel scenario. The covariance $R_{i j}$ expresses the amount of power which is commonly radiated or received by the antennas $i$ and $j$ and which can not be distinguished anymore in the receiver signals. The goal is to minimize the covariance $R_{i j}$ which can only be done on antenna side. For the stochastic channel model, the covariance follows Eq. (8).

$$
\begin{gathered}
R_{i j} \approx \int_{0}^{2 \pi} \int_{0}^{\pi}\left(C_{\vartheta_{i}}(\vartheta, \varphi) \cdot C_{\vartheta_{j}}^{*}(\vartheta, \varphi)+X P R \cdot C_{\varphi_{i}}(\vartheta, \varphi)\right. \\
\left.\ldots C_{\varphi_{j}}^{*}(\vartheta, \varphi)\right) \cdot p_{\vartheta, \varphi}(\vartheta, \varphi) \cdot e^{j k r_{i j}} \cdot \sin (\vartheta) d \vartheta d \varphi
\end{gathered}
$$

The expression contents the far field patterns $C$ for each antenna $i$ in an array in $\vartheta$ and $\varphi$ polarization in dependence of the azimuthal and elevational directions. The patterns of the antenna elements $i$ and $j$ are multiplied for each polarization direction and can be interpreted as the commonly radiated part of the power in one spatial direction. The crosspolarization ratio $X P R$ is a property of the propagation channel and is a measure for the conversion of a linearly polarized wave into its orthogonal polarization. If the ratio is $X P R=1$, the power of a linearly polarized wave is equally distributed into its co- and cross polarization direction. This is generally assumed in indoor WLAN channels and can be explained by the numerous reflections in the propagation pathes. The product of the patterns is further on multiplied by the propability density function emphasizing the spatial direction of the dominant propagation pathes. Another factor is the exponential term expressing the phase delay between the antenna signals in a specific room direction. As mentioned before it is important to minimize the the covariance between each antenna element in an antenna array for MIMO applications. Three antenna parameters out of Eq. (8) can be tuned to achieve this goal. One parameter is the pattern diversity. If the antennas "look" in different directions the product of the patterns is minimized. Another approach is separating the antennas in order to benefit from the phase difference between the signals. The final possibility is to use antennas with different polarizations. In this case, the pattern product can be minimized. If this condition can be kept over a large angular spread, the correlation is small in many channel scenarios.

It was shown, how the LOS and the NLOS components of the channel matrix $\tilde{\mathbf{H}}$ can be minimized. In both cases it can be pointed out that polarization diversity helps to exploit the spatial diversity of the channel. This statement has been proven by measured channel matrices for different antenna setups as described in the following section.

\section{Measurement}

The measurement of MIMO channel matrices in WLAN channels requires a possibility for simultaneously transmitting and receiving RF signals in both frequency bands around $2.4 \mathrm{GHz}$ and $5.4 \mathrm{GHz}$. The equipment used and the applied measurement method is explained in Sect. 3.1. The Sect. 3.1.1 shortly introduces the logarithmic periodic antenna used for the measurement. The measurement results taken in the office environment are described in Sect. 3.1.2 are shown and discussed in Sect. 3.2.

\subsection{Measurement hardware}

The HaLo220 system consists of two router like devices equipped with antennas and connected to a PC via USB as shown in Fig. 3. Each device contents two dual band transceiver units that can be configured as receiver or transmitter. A fast memory allows the playback or the acquisition of baseband data with different bandwidths up to $40 \mathrm{MHz}$. The synchronous transmission and reception of data makes it possible to measure the complex impulse response of the channel. However it is necessary to estimate the CIR (channel impulse response) for each path of the $2 \times 2$ system by considering the restrictions of the coherence time and bandwidth from Sect. 2. The method applied to separate the single pathes is an evaluation of the transmission of two $\mathrm{CW}$ signals with a slight frequency shift $\Delta f$ of $200 \mathrm{kHz}$. The receiver starts recording with a sample rate of $f_{s}=5$ MSamples/s, when the signal level crosses an adjustable trigger level as the $\mathrm{CW}$ signals impinge. After acquisition, $n=50000$ samples of the received complex baseband data are transferred to the PC where an FFT is applied. The duration of the transmission is $t_{t r}=10 \mathrm{~ms}=n / f_{s}$, which lies within the coherence time interval of $18 \mathrm{~ms}$ according to Sect. 2.1. The FFT for each of the two receiver signals shows two peaks 
with different magnitudes and angles according to the transmitted $\mathrm{CW}$ signals. By determining these peaks and their values the channel matrix can be composed for every coherence time and the channel capacity can be calculated according to Eq. (4). Because of the flat fading channel the complex values in the frequency domain result in a delta peak in the time domain with the same value. To get trustable predictions of the channel behavior, 1000 measurements for each channel scenario have been taken.

\subsubsection{Antenna hardware}

To demonstrate the influence of polarization diversity on the examined channels two different types of antennas were used. The first type is the well known $\lambda / 4$ monopole that can be mounted on the HaLo system directly. Two of these elements were used in a distance of about $\lambda$ for $f=2.4 \mathrm{GHz}$ and were both aligned vertically. This setup offers weak polarization diversity. In contrast, the logarithmic periodical antenna shown in Fig. 4 provides the radiation of two linearly polarized waves with high $A R$. This planar antenna type is a self-complementary structure applied on $F R 4$ substrate consisting of four orthogonally placed arms as shown in Fig. 4 (Klemp and Eul, 2006). It has originally been designed for broadband applications. Exciting an opposite pair of arms with a differential signal generates a linear polarized wave radiating normally to the substrate plane in both directions. The feeding points for each element are in the center of the antenna and are connected via a semirigid coaxial waveguide from the backside. To reduce interactions with the coaxial cables, the feeding network and the generation of mantle modes a $\lambda / 4$ reflector is placed on the backside of the antenna. Hence, the gain in main beam direction is increased to approximately $G=5 \mathrm{dBi}$ for a narrow band around the center frequency in a full wave simulation at $2.4 \mathrm{GHz}$. To switch between the two measurement frequencies the distance of the reflector can be adjusted. In this configuration, the simulated $A R$ of the orthogonal arm pairs is better than $30 \mathrm{~dB}$ for both frequencies.

\subsubsection{Office scenario}

A floor plan of the examined office environment is illustrated in Fig. 5. The transmit and receive antennas have been placed in the same height of about $1.2 \mathrm{~m}$ above the ground and in a distance of $5 \mathrm{~m}$. To separate between a channel which is dominated by the LOS component and the NLOS component, respectively, a metal plate is placed in front of the transmit antennas. The plate causes a reflection of the transmitted signal to the back of the room. To avoid the direct backscattering from the plate back into the LP antenna it was twisted a little bit (see the left image in Fig. 5). In the monopole setup the LP antennas were just replaced by two vertically oriented monopoles at the same place that are directly connected to the HaLo housing. Three different an-

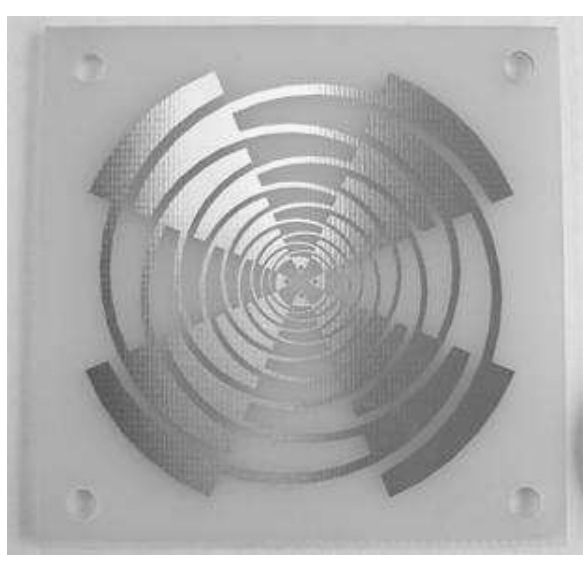

Fig. 4. Logarithmically periodic antenna.

tenna setups were examined. In two cases similar antenna types were used on transmitter and receiver side, monopole and LP antennas respectively. In the third case two different types of antennas were used, an LP antenna on receiver side and two monopoles on transmitter side.

\subsection{Measurement results}

The measurement results are presented as a cumulative distribution function (cdf) of the measured channel capacities for 1000 coherence time intervals. The cdf can be interpreted as the probability of the channel capacity to fall below the value on the abscissa. It is also called the outage rate. Figure 6 shows the results for the LOS scenario (without metal plate) for the two subchannels for each of the three antenna configurations. In the case of using two LP antennas both subchannels are strong. The curves show a high slope because of the dominating static LOS component of the channel. There is no significant change in the channel capacity and due to the good polarization decoupling the channel matrix has high rank which results in a good quality of both subchannels. In comparison to the other antenna setups the sum capacity of the $L P \times L P$ case is the best. Replacing the transmit antenna with two vertically oriented monopoles causes a decrease of the stronger subchannel due to the missing gain into the direction of the receiving antenna. The influence on the weaker subchannel is more significant. It decreases from about $10 \mathrm{bit} / \mathrm{s} / \mathrm{Hz}$ to $2.5 \mathrm{bit} / \mathrm{s} / \mathrm{Hz}$ at an outage rate of -1 . Both signals are transmitted with vertical polarization and will be received by the vertical polarized arm pair of the LP antenna with more signal power than by the horizontally polarized arm pair. By means of the channel matrix the elements in the second column are much lower than the ones in the first column. The subchannels can hardly be seperated in this case. Most of the signal power received in the horizontal direction is caused by reflections on the walls which avoids the weaker subchannel to vanish completely. This relation results from the smaller slope of the weak compared to the 


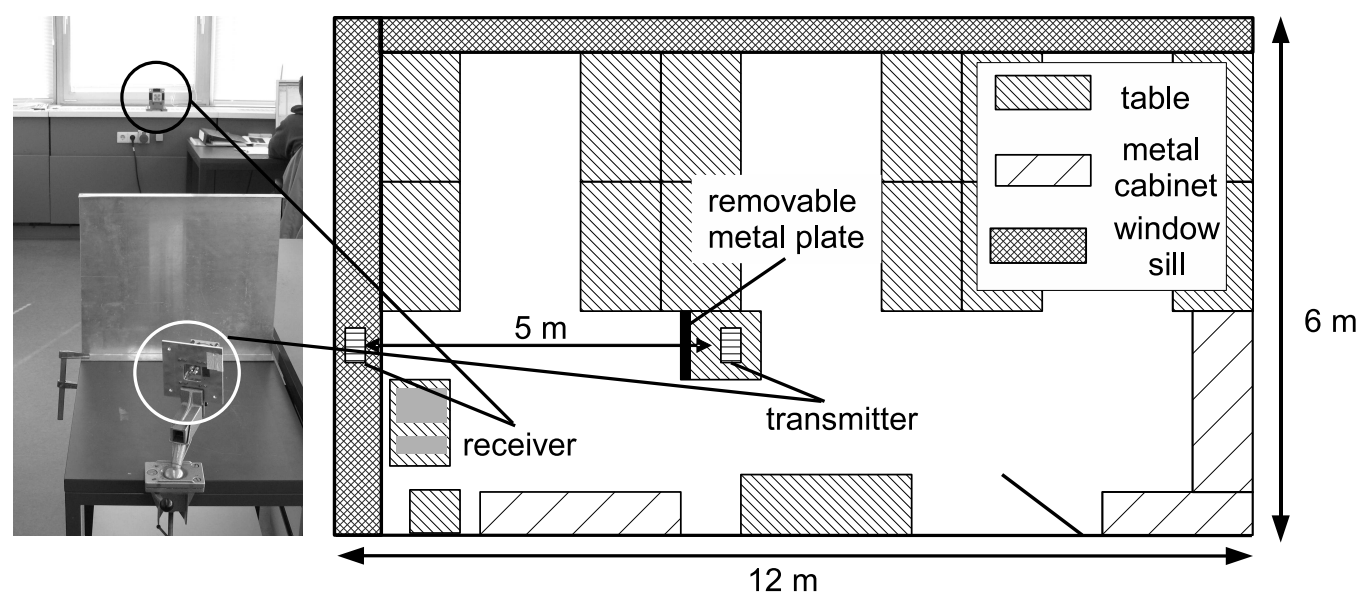

Fig. 5. Floor plan of the office room.

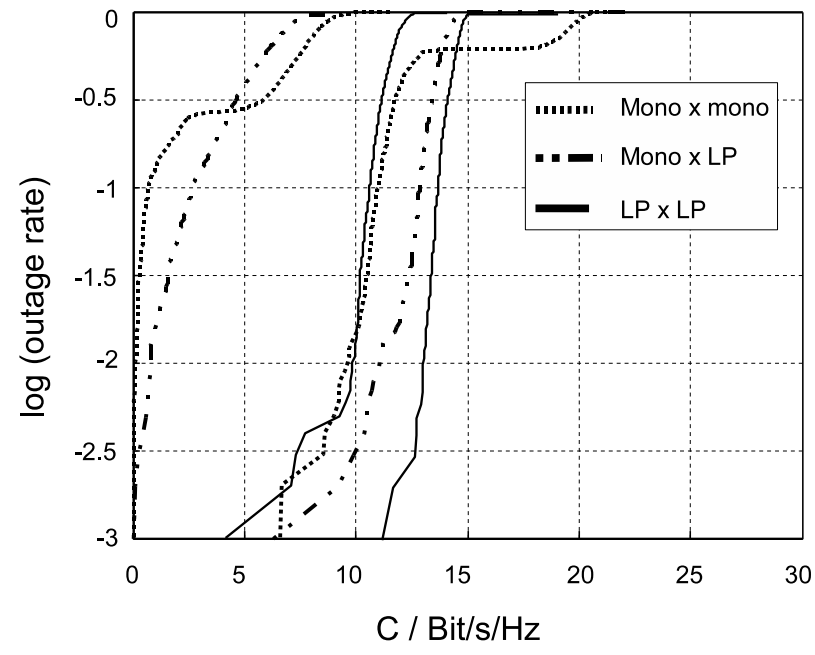

Fig. 6. Measured cdf of the subchannels at $2.4 \mathrm{GHz}$ for three antenna setups in the LOS scenario.

strong subchannel which implies a higher weight of rayleigh fading in the channel according to a decreasing rice factor $K$ in Eq. (5). In the last antenna setup with monopole antennas on transmit and receiver side this effect gets even stronger. The very constant far field pattern of the single antenna elements in azimuthal direction leads to multiple transmission pathes that are frequently changing. The weaker LOS path results in a SNR loss which can be seen by the vertically shifted curve of the stronger subchannel with and without LP antenna. The higher influence of the stochastic properties of the channel results in a higher spread of the measured channel capacities.

Similar results can be obtained by the measurement at $5.4 \mathrm{GHz}$ in Fig. 7. All mentionable effects can be shown regarding the setups of two LPs and two monopoles. In comparison to the measurement at $2.4 \mathrm{GHz}$ the curves are shifted

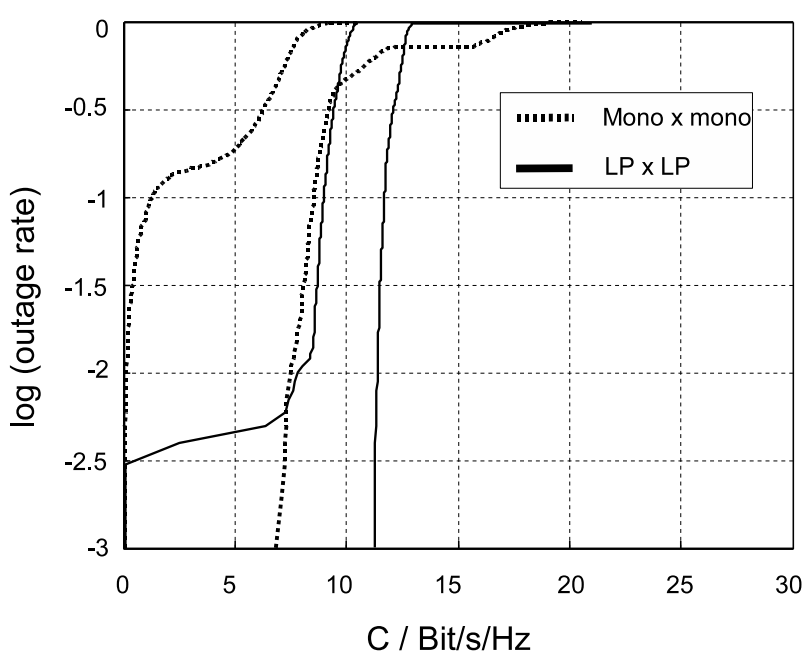

Fig. 7. Measured cdf of the subchannels at $5.4 \mathrm{GHz}$ for two antenna setups.

horizontally because of the higher path loss. The shape of the curves, however, is kept as the propagation properties seem to be similar. The subchannels of the LOS dominated channel in the $L P \times L P$ setup is expressed by the high slope of the curves. Both subchannels are strong due to the polarization decoupling which drops in the case of two monopole antennas on each side. Here again the stochastic influences of the channel decrease the slope of the curves. Up to now it can be pointed out that polarization diversity leads to a better decoupling of the subchannels for both frequencies in the LOS case. The good SNR of the channel is due to the gain in main beam direction resulting in a high capacity of both subchannels.

The next step is to analyze the results for the NLOS case. According to Sect. 2.3.2 polarization diversity will lead to 


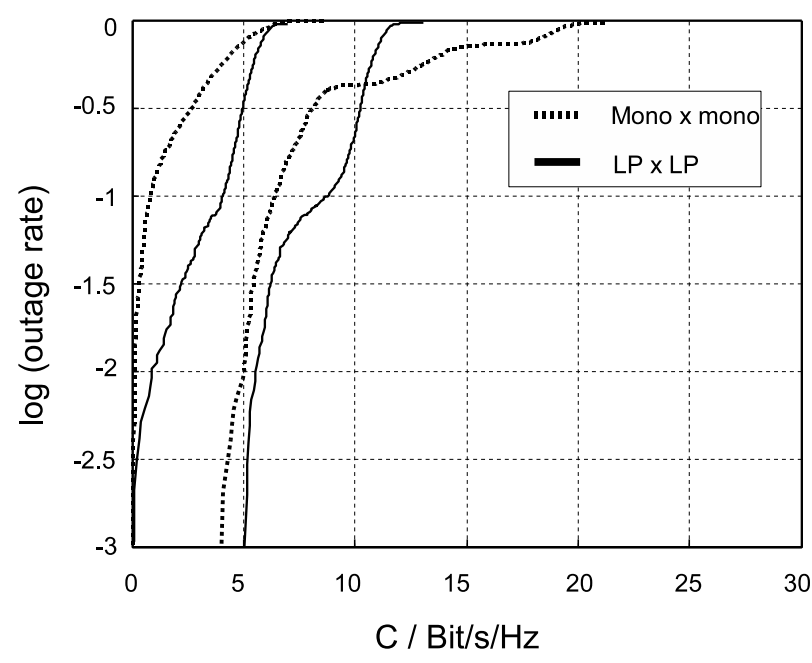

Fig. 8. Measured cdf of the subchannels at $5.4 \mathrm{GHz}$ for two antenna setups in the NLOS scenario.

small correlation of the antenna elements and therefore to a decoupling of the subchannels. The comparison of the subchannel capacities for the setup of two LP antennas and four monopole antennas for $5.4 \mathrm{GHz}$ are shown in Fig. 8. For both antenna setups the capacities are smaller compared to the ones obtained in the LOS scenarios because of the missing LOS component which has been faded out by placing the metal plate in front of the transmit antennas. Starting with the LP antenna setup it can be determined that the smaller subchannel is not that strong anymore in comparison to one in the LOS case. This is due to the depolarization effect of the channel considered in expression (8) with the variable $X P R$. The slope of the curves is decreased because of the changing channel conditions as already seen in the changing weight of the Rice factor between the monopole and the LP setup in the LOS case. In the NLOS case the stronger subchannels are similar except for the slope. It seems that in the monopole setup the propagation pathes are more fluctuating than in the LP channel. Although this property would lead to a decorrelation in conjunction with the distance of about $\lambda$ for the monopoles it can be pointed out that the weaker subchannel of the LP setup is still better than in the monpole setup. This emphasizes the role of polarization decoupling in the NLOS case.

\section{Conclusions}

The contribution highlighted the antenna properties leading to a good MIMO performance for LOS as well as for NLOS channels assuming a stochastic channel model and a narrow band propagation channel. It has been determined that polarization diversity can improve orthogonality of the subchannels. This issue has been investigated using different antenna setups with high and low polarization decoupling. The LP antenna offers a high depolarization and leads to a good performance compared to a monopole setup that is used in the most present WLAN devices. In future WLAN applications even more antennas will have to be placed in order to exploit the spatial diversity properties of the channel. Due to the unaltered geometrical restrictions antenna designers will be forced to consider every possibility to decrease the correlation between the single antenna elements. Applying planar antennas with different polarization and pattern properties can be a solution of this problem as presented in this paper.

\section{References}

Foschini, G. J. and Gans, M. J.: On Limits of Wireless Communications in a Fading Environment when Using Multiple Antennas, Wireless Personal Communications, 6, 311-335, 1998.

Fujimoto, K. and James, J. R.: Mobile antenna systems handbook, Artech House, 2001.

Jiménez, V. P. G.: Channel Estimation for Bit-loading in OFDMbased WLAN, University Carlos III of Madrid, Spain, 2002.

Klemp, O. and Eul, H.: On the Application of Multimode Diversity Reception using Miniaturized Wideband Log.-Per, Planar Antennas Proceedings of the 9th European Conference on Wireless Technology, 2006.

McDonnell, J. T. E., Spiller, T. P., and Wilkinson, T. A.: RMS Delay Spread in Indoor LOS Environments at $5.2 \mathrm{GHz}$, HP Laboratories Bristol, 1998.

Rappaport, T. S.: Wireless Communications: Principles and Practice, Prentice Hall PTR, 1996.

Schumacher, L. and Kermoal, J. P.: A Stochastic MIMO Radio Channel Model With Experimental Validation, IEEE J. selected areas in communications, 20(6), 1211-1226, 2002.

Sklar, B.: Rayleigh Fading Channels in Mobile Digital Communication System Part 1: Characterization, IEEE Communications Magazine, 1997. 